\title{
Surface Layering in Liquid Gallium: An X-Ray Reflectivity Study
}

\section{Citation}

Regan, M. J., E. H. Kawamoto, S. Lee, and Peter S. Pershan, N. Maskil, M. Deutsch, O. M. Magnussen, B. M. Ocko, and L. E. Berman. 1995. Surface layering in liquid gallium: An x-ray reflectivity study. Physical Review Letters 75(13): 2498-2501.

\section{Published Version}

doi:10.1103/PhysRevLett.75.2498

\section{Permanent link}

http://nrs.harvard.edu/urn-3:HUL.InstRepos:10357490

\section{Terms of Use}

This article was downloaded from Harvard University's DASH repository, and is made available under the terms and conditions applicable to Other Posted Material, as set forth at http:// nrs.harvard.edu/urn-3:HUL.InstRepos:dash.current.terms-of-use\#LAA

\section{Share Your Story}

The Harvard community has made this article openly available.

Please share how this access benefits you. Submit a story.

\section{Accessibility}




\title{
Surface Layering in Liquid Gallium: An X-Ray Reflectivity Study
}

\author{
M. J. Regan, E. H. Kawamoto, ${ }^{*}$ S. Lee, ${ }^{\dagger}$ and P. S. Pershan \\ Division of Applied Sciences and Department of Physics, Harvard University, Cambridge, Massachusetts 02138 \\ N. Maskil and M. Deutsch \\ Department of Physics, Bar-Ilan University, Ramat-Gan 52100, Israel \\ O. M. Magnussen and B. M. Ocko \\ Department of Physics, Brookhaven National Laboratory, Upton, New York 11973 \\ L. E. Berman \\ National Synchrotron Light Source, Brookhaven National Laboratory, Upton, New York 11973
}

(Received 10 March 1995)

\begin{abstract}
Surface-induced atomic layering in liquid gallium has been observed using x-ray reflectivity, ultrahigh vacuum conditions, and sputtered clean surfaces. Reflectivity data, collected on a supercooled liquid sample to momentum transfers as large as $q_{z}=3.0 \AA^{-1}$, exhibit a strong maximum near $2.4 \AA^{-1}$ indicating a layer spacing that is comparable to its atomic dimensions. The amplitude of the electron density oscillations decays with a characteristic length of $6 \AA$. This is unexpectedly twice that of recent results for $\mathrm{Hg}$, and the difference may be related to covalent bonding or supercooling.

PACS numbers: 61.10.-i, 61.25.Mv, 68.10.-m
\end{abstract}

The similarity of the bulk structure for both metallic and nonmetallic liquids obscures fundamental differences in the basic physics of the two. The structure of nonmetallic liquids can be understood in terms of classical pairwise interactions; however, for metals, Coulombic interactions couple the quantum electron gas and the classical ions leading to interatomic interactions that strongly depend on density $[1,2]$. The strong density variation associated with the free surface provides a natural and convenient way of testing this aspect of the theory [3-6]. This was realized early by Rice and co-workers, who proposed to study the surface density profile directly by x-ray reflectivity measurements $[3,7]$. The problem of surface structure has attracted considerable attention and there are now a number of theoretical, both analytic [4-6] and molecular simulation [8,9], and experimental studies [10-12] of liquid metal surfaces. One of the more interesting ideas generated from these efforts is that partial delocalization of the near surface conduction electrons suppresses positional fluctuations of the near surface ion cores. A direct consequence of this is the prediction of atomic layering at the surface of liquid metals, in contrast to nonmetallic liquids.

The most convenient elements for liquid metal experiments are $\mathrm{Ga}$ and $\mathrm{Hg}$, since both are liquid at relatively low temperatures. Earlier experimental efforts to search for surface-induced layering were inconclusive [13,14], and it is only very recently that Magnussen et al. [15] were able to unambiguously demonstrate layering at the surface of liquid Hg. Gallium, on the other hand, exhibits several unique qualities which distinguish it from $\mathrm{Hg}$ and most other liquid metals. Perhaps the most striking of these is the asymmetry in the first peak of the bulk liquid structure factor [16], which is not observed in typical liquid metals or $\mathrm{Hg}$ and which has been attributed to a relatively high degree of covalent bonding, leading to orientational correlations in bulk liquid Ga $[17,18]$. This is probably related to other unusual properties of $\mathrm{Ga}$, with respect to most other metals, such as an exceptionally large liquid range $\left(\sim 2000^{\circ} \mathrm{C}\right)$, a large crystalline unit cell, and anomalous expansion upon solidification. Competition between the covalent and metallic bonding could explain the observed differences between the surface order for Ga [see Fig. 1(c) below] and that of $\mathrm{Hg}$ [15]; however, the microscopic origin of these differences is not yet clear, and, from this point of view, it is critical to understand surface layering for a variety of liquid metals. Finally, in comparison with $\mathrm{Hg}, \mathrm{Ga}$ has a higher surface tension, leading to mean square thermal fluctuations (capillary waves) in the local height of the surface that are only $70 \%$ of that of $\mathrm{Hg}$.

Direct observation of atomic layering requires that specular reflectivity measurements be extended to a wavevector transfer $q_{z} \approx 2 \pi / d \sim 2-2.5 \AA^{-1}$, where $d$ is of the order of the atomic diameter, 2.5-3.0 $\AA$. Here we report the results of an x-ray reflectivity study from the liquid Ga surface under ultrahigh vacuum (UHV) which shows the existence of a quasi Bragg peak at $q_{z}=$ $2.4 \AA^{-1}$ (Fig. 1). This is a clear indication of surfaceinduced atomic layering. Although different models can be constructed to agree with the reflectivity data, the unique feature that must be present in each of these is a layered electron-density profile with a surface-induced layer spacing similar to the $\mathrm{Ga}$ atomic dimensions and with the layering extending an exponential decay length of $\sim 3$ atomic diameters into the bulk [Fig. 1(d)].

The reflectivity data were collected on a shallow supercooled $\left(\sim 22^{\circ} \mathrm{C}\right.$, melting point at $\left.29.8^{\circ} \mathrm{C}\right)$ liquid $\mathrm{Ga}$ 


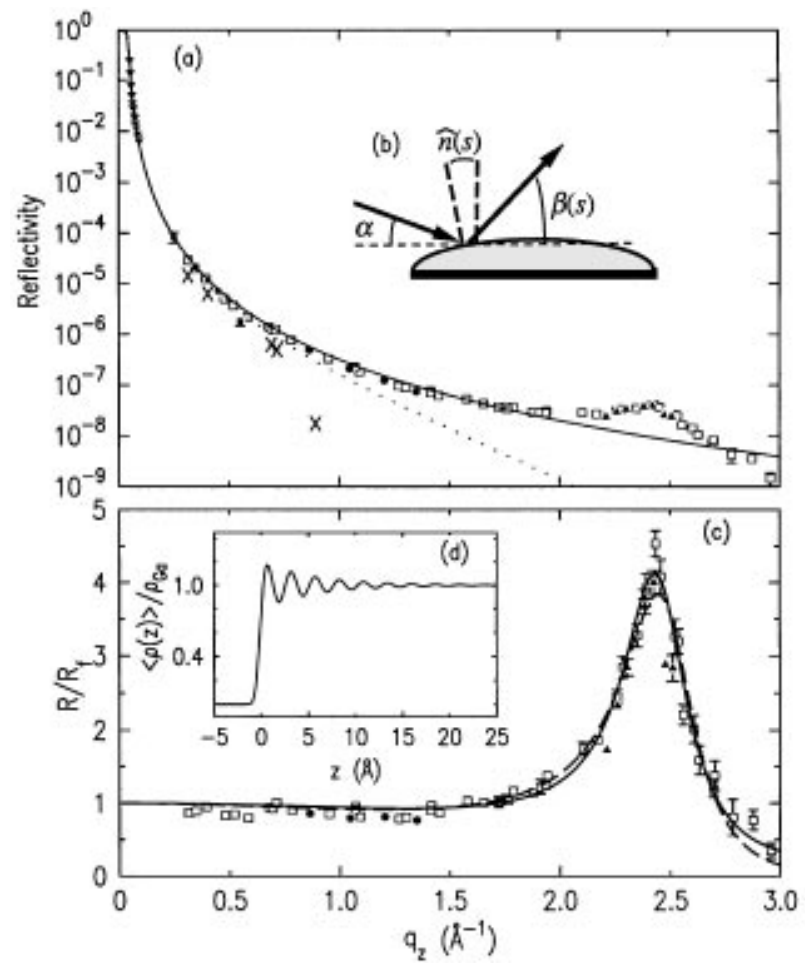

FIG. 1. (a) Measured reflectivity curve for liquid Ga. Data marked with an $\times$ were collected prior to the sample cleaning procedure; - collected during the sputter cleaning procedure; $\square$ from integrating the $\beta$ scans on a clean surface; $\boldsymbol{\Delta}$ from a relative comparison with $2 \theta$ scans; $\boldsymbol{\nabla}$ from the low-angle method described in Ref. [14]; $\square$ are data from Ref. [14]. The Fresnel reflectivity is denoted by a solid line, and the dotted line is the Fresnel model convoluted with the combination of a $0.82 \AA$ rough capillary wave and the $\mathrm{Ga}$ atomic scattering factor. (b) Schematic diagram of curved liquid-surface kinematics (curvature exaggerated for clarity). (c) Reflectivity normalized by the Fresnel reflectivity. The best fit by the exponentially decaying sine model is a solid line, and the dashed line is the fit by the distorted crystalline model. (d) Corresponding electron density profiles, which are indistinguishable in the figure for the two models.

film supported by a $32 \mathrm{~mm}$ diameter Mo substrate. The thin layer $(\sim 0.2 \mathrm{~mm}$ thick) is necessary for suppression of mechanically excited surface waves by viscous drag at the $\mathrm{Ga} / \mathrm{Mo}$ interface. The sample was prepared by sputter cleaning the Mo surface for 30-45 $\mathrm{min}$ in a dc glow discharge of Ar and then dropping the liquid $\mathrm{Ga}$ onto the glowing Mo. Although contact angles as small as $\sim 10^{\circ}$ (as judged by the eye) were possible, the large surface tension for Ga leads to a curved surface, with the measured radius of curvature at the top of the drop on the order of $800 \mathrm{~mm}$. The samples were then frozen in a nitrogen environment, transported to the National Synchrotron Light Source (NSLS), melted, and placed into the UHV chamber where x-ray measurements were made at $\mathrm{O}_{2}$ partial pressures less than $10^{-11}$ Torr. Surface oxides that form during transport, when the samples are exposed to air, are removed by sputtering with 2-keV Ar ions. This approach differs substantially from measurements on $\mathrm{Hg}$ [15], which are simplified by its relatively small reduction potential and consequently allows the surface to be kept oxide free by enclosing it in a reducing atmosphere of $\mathrm{H}_{2}$ gas. For most other metals this is not possible, and surface studies will require UHV techniques.

The data were collected at the wiggler beam line X-25 at the NSLS using a toroidal mirror and a liquid reflectometer with a water-cooled $\mathrm{Ge}(111)$ crystal monochromator set to reflect at $\lambda=0.6532 \AA$. Figure 1(b) illustrates the kinematics of the experiment. The angle of the beam with respect to the horizontal, $\alpha$, was varied by tilting the monochromator, and the position where the beam strikes the sample was varied by adjusting the vertical position of the sample, $s$. For a curved sample surface and a given $\alpha\left(\delta \alpha \approx 0.006^{\circ}\right)$, the local surface normal $\hat{n}(s)$ determines the angle of incidence relative to the surface as well as the angle of reflection $\beta(s)$; the extent of the illuminated area determines the divergence $\delta \beta(s)$ of the reflected beam. Careful mapping of $\beta(s)$ and $\delta \beta(s)$ vs $s$ allows a determination of the local and average sample curvature [14,19].

In Figs. 2(a) and 2(b) we show typical profiles of the reflected beam as a function of $\beta$ (in the plane of reflection) measured for $\alpha=2.33^{\circ}$ and $6.9^{\circ}$. For sufficiently small $\alpha$, the reflected signal is large and easy to separate from the diffuse scattering. For larger $\alpha$, near the peak in the bulk liquid structure factor, the diffuse scattering is considerably larger than the reflected signal; however, they can be separated by making use of their measured line shapes [19]. For a given $\alpha$ and $s$, the reflectivity $R\left(q_{z}\right)$ at $q_{z}=(4 \pi / \lambda) \sin [(\alpha+\beta(s)) / 2]$ is determined by integration over $\beta$ after removal of the diffuse scattering. Comparison of reflected intensities for different $\alpha, s$, and $\beta(s)$ established that $R\left(q_{z}\right)$ is insensitive to the precise location of illuminated surface area. With this approach, the reflectivity is obtained by measuring within the plane of reflection; a supplementary mode of measurement entails scanning the detector across the plane of reflection (i.e., a $2 \theta$ scan). Examples of $2 \theta$ scans [Figs. 2(c) and 2(d)] show the reflected profiles as resolution limited peaks above an essentially constant background.

The reflectivity obtained from analysis of both the $\beta$ and $2 \theta$ scans are plotted in Figs. 1(a) and 1(c), and there is good agreement. The importance of in situ surface cleaning is clearly demonstrated by inclusion in the figure of data collected on oxidized Ga surfaces and then on sputtered clean surfaces, as well as during the first $\sim 2-3 \mathrm{~h}$ into the sputtering procedure. Also included in Fig. 1(a) are previous measurements from our group for $q_{z} \leq 0.55 \AA^{-1}$ [14], the theoretical Fresnel reflectivity $\left(R_{F}\right)$ and that expected for a monotonic density profile with the theoretically predicted capillary wave roughness of $0.82 \AA$. The data obtained on the clean Ga show 

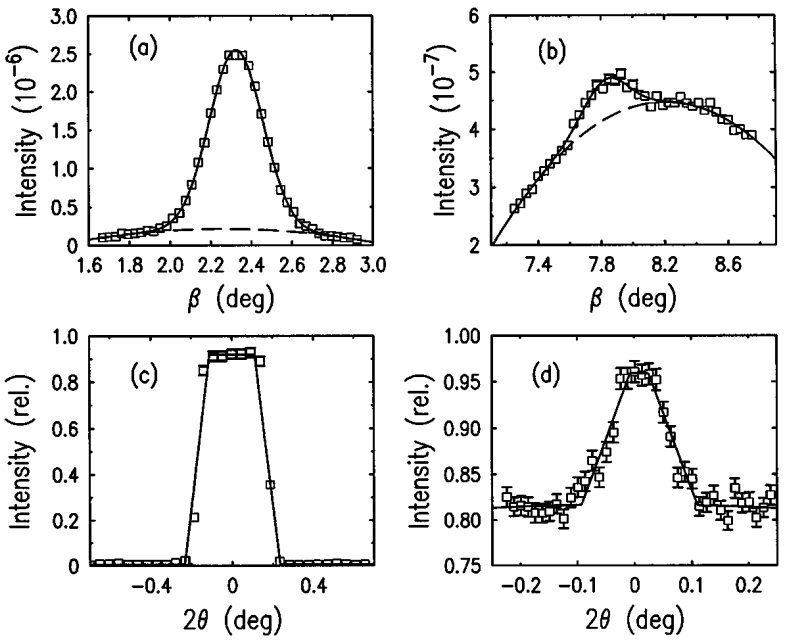

FIG. 2. Scans of intensity in the plane of reflection (scan $\beta$; $2 \theta=0)$ for (a) $\alpha=2.33^{\circ}\left(q_{z}=0.782 \AA^{-1}\right)$ and (b) $\alpha=6.9^{\circ}$ $\left(q_{z}=2.48 \AA^{-1}\right)$. Dashed lines are fits to the diffuse scattering, and solid lines are fits to the reflection superimposed on the diffuse scattering. Corresponding scans through the plane of reflection (scan $2 \theta ; \beta$ set at the specular reflection), for (c) $\alpha=2.33^{\circ}$ and (d) $\alpha=6.9^{\circ}$, illustrate a second method to distinguish the reflection. To compensate for the decrease in reflectivity for $\alpha=6.9^{\circ}$, the height of the incident beam was approximately $2 \times$ greater than for $\alpha=2.33^{\circ}$. The horizontal detector resolution was set at $0.4^{\circ}$ in (c) and $0.1^{\circ}$ in (d). Confidence limits for these fits are included in the error bars shown in Fig. 1.

no appreciable deviation from Fresnel theory except for $q_{z}>2.0 \AA^{-1}$, where a well-defined maximum is evident. The marked difference in reflectivity from a dirty surface, which deviates greatly from $R_{F}$ and was not measurable for $q_{z}>1 \AA^{-1}$, and then from a clean one, highlights the importance of maintaining a UHV-clean, oxide-free surface for these studies.

When scaled by the Fresnel reflectivity [Fig. 1(c)], the data are of a rather simple form. The ratio $R / R_{F}$ can be generally described with as few as four parameters in real space that are equivalent to the amplitude, decay length, and spacing of the electron density oscillations into the bulk liquid and the interfacial roughness. Although a number of density models can be constructed with additional parameters that lead to even better agreement with the data, only two of the simplest models will be mentioned here. Without additional data at $q_{z} \geq$ $3.0 \AA^{-1}$, it is not possible to determine much more than the basic features of the layering profile.

For $q_{z}$ larger than 4-5 times the critical wave vector, $R / R_{F}$ is related to the average electron density along the surface normal, $\langle\rho(z)\rangle$, by [20]

$$
\frac{R\left(q_{z}\right)}{R_{F}\left(q_{z}\right)} \approx\left|\frac{1}{\rho_{\infty}} \int\left[\frac{d\langle\rho(z)\rangle}{d z}\right] e^{i q_{z} z} d z\right|^{2},
$$

with $\rho_{\infty}$ the known bulk electron density. The simplest layering profile that can be constructed, similar to previous liquid crystal models, is based on an error-function interfacial profile (width $\sigma$ and offset by $z_{0}$ ) modulated by an exponentially decaying sine wave:

$\langle\rho(z)\rangle / \rho_{\infty}=\operatorname{erf}\left[\left(z-z_{0}\right) / \sigma\right]+\theta(z) A \sin (2 \pi z / d) e^{-z / \xi}$.

$\theta(z)$ is the step function, $d$ the interlayer spacing, $\xi$ the exponential decay length, and $A$ an amplitude. Fits by this model are shown in Fig. 1(c) (solid line), with $d=2.56 \pm 0.01 \AA, \quad \xi=5.8 \pm 0.4 \AA$, $A=0.20 \pm 0.02, \quad \sigma=0.50 \pm 0.04 \AA, \quad$ and $z_{0}=-0.24 \pm 0.06 \AA$. The electron density profile is shown in Fig. 1(d); profiles computed within the errors of the parameters would be indistinguishable in the figure.

Similar results are obtained with the distorted crystal model that has been used for liquid $\mathrm{Hg}$ [15]. In this model, the root-mean displacement of the $n$th layer, $\sigma_{n}$, increases with depth into the bulk liquid. To simplify and limit the number of parameters, let $\sigma_{n}^{2} \approx \sigma_{0}^{2}+n \bar{\sigma}^{2}$ with $\bar{\sigma}$ a measure of the increasing root-mean displacement as the density approaches the bulk liquid and $\sigma_{0}$ a displacement common to each layer. With the spacing $d$ between layers fixed, the reflectivity is

$$
\frac{R\left(q_{z}\right)}{R_{F}\left(q_{z}\right)}=e^{-q_{z}^{2} \sigma_{0}^{2}}\left|\frac{f\left(q_{z}\right) q_{z} d}{Z}\right|^{2}\left|e^{q_{z}^{2} \bar{\sigma}^{2}} e^{-i q_{z} d}-1\right|^{-2}
$$

where $f\left(q_{z}\right)$ is the $\mathrm{Ga}$ atomic scattering factor [atomic dispersion corrections $f^{\prime}\left(q_{z}\right)$ and $f^{\prime \prime}\left(q_{z}\right)$ are negligible] and $Z$ the atomic number. Figure 1(c) shows the best fit (dashed line) of this model, with $d=2.50 \pm 0.01 \AA$, $\sigma_{0}=0.67 \pm 0.01 \AA$, and $\bar{\sigma}=0.40 \pm 0.01 \AA$.

Analysis with either model leads to essentially the same results. The interlayer spacing $d$ is $\sim 10 \%$ less than the near neighbor spacing in the bulk liquid, which is expected from the stacking of neighboring layers. For the second model, in particular, the electron density profile can be interpreted as a local structure that is broadened by thermally induced capillary waves, denoted by a width $\sigma_{\mathrm{cw}}$ [21]. Given the Ga surface tension at the melting point $\gamma=0.718 \mathrm{~N} / \mathrm{m}$, atomic diameter $\sim 2.5 \AA$, and an experimental resolution of $0.062 \times 0.0008 q_{z} \AA^{2}$, calculated values for $\sigma_{\mathrm{cw}}$ range from 0.75 to $0.90 \AA$ over the measured $q_{z}$ range. These values are in agreement with the width of the top layer, which in the second model is given directly by $\sqrt{\sigma_{0}^{2}+\bar{\sigma}^{2}}=0.78 \AA$, and indicate that the liquid metal surface is extremely flat with no measurable roughness except for the broadening expected from capillary wave theory.

The layering extends into the bulk liquid with an exponential decay length of $5.8 \pm 0.4 \AA$, which corresponds to $\sim 3$ atomic diameters. This is approximately twice the decay length that has been measured for $\mathrm{Hg}$ at room temperature and, although the origin of the difference is not understood, it may result from either a supercooled $\mathrm{Ga}$ sample or basic differences in the surface properties of 
liquid $\mathrm{Ga}$ and $\mathrm{Hg}$, which are, for example, manifested by the much smaller vapor pressure and larger surface tension of Ga. Another possibility is the idea mentioned earlier about the importance of different orientational correlations in the near neighbor packing around individual $\mathrm{Ga}$ and $\mathrm{Hg}$ atoms.

Furthermore, unlike $\mathrm{Hg}$, the decay in layering amplitude for Ga is not well characterized by the decay present in its bulk pair correlation function. Since the correspondence for $\mathrm{Hg}$ was taken to indicate that the layering is dominated essentially by the same isotropic short-range effective interactions which determine the bulk packing [15], its absence here may be related to other interactions at the Ga surface, e.g., bond correlations, which are possibly more dominant in the near surface region. Another subtle difference between the $\mathrm{Ga}$ and $\mathrm{Hg}$ results is that, in agreement with the Ga simulations [9], we find no evidence for the broad tail on the vapor side of the interface that was experimentally observed [15] and predicted by simulations [22] for the surface of Hg. Finally, we note that our results are in agreement with computer simulations on $\mathrm{Ga}$, which predict a similarly layered profile that extends $\sim 3$ atomic diameters into the bulk [9].

The excess lamellar order that is observed here is likely related to the excess order on the liquid side of the liquid/crystal interface that Turnbull [23] and, more recently, Spaepen [24] have discussed in connection with the supercooling of liquid metals. It is, therefore, worth comparing the two phenomena. One important issue is the amount by which surface-induced lamellar order may possibly be supplemented by in-plane positional order (i.e., in the surface plane). It is not clear whether lamellar order alone can fully account for the negative surface entropy at the liquid/ crystal interface, since the crystal lattice may induce inplane order in the liquid. At the Ga liquid/vapor interface, computer simulations and grazing-incidence x-ray experiments indicate that the in-plane structure is similar to the bulk liquid without any obvious positional order [25], although this needs to be investigated further. The subject is also closely related to premelting phenomena in which certain crystalline facets exhibit excess disorder at the liquid/crystal interface, leading to a positive surface entropy. Although it is important to understand the microscopic origin of these differences and there have been major efforts to understand, for example, the theory of premelting [26], structural data on the excess order at liquid interfaces mentioned remain also nonexistent.

This work has been supported by grants from the U.S. Department of Energy, No. DE-FG02-88-ER45379, and the National Science Foundation, No. DMR-89-20490 and No. DMR-91-13782. Brookhaven National Laboratory is supported by DOE Contract No. DE-AC0276CH00016. M. D. acknowledges support by the Bar-Ilan Research Authority. S. L. acknowledges support from the Pohang Light Source, Pohang, Korea.

*Present address: Department of Physics and Astronomy, University of Massachusetts, Amherst, MA 01003.

†Present address: Department of Physics, Ajou University, Suwon 442-749, Korea.

[1] P. Hohenberg and W. Kohn, Phys. Rev. 136, B864 (1964).

[2] W. Kohn and L. J. Sham, Phys. Rev. 140, A1133 (1965).

[3] S. A. Rice, Proc. Natl. Acad. Sci. U.S.A. 84, 4709 (1987).

[4] M. A. Gómez and E. Chacón, Phys. Rev. B 46, 723 (1992).

[5] R. Evans et al., Mol. Phys. 76, 1 (1993).

[6] M. Iwamatsu and S. K. Lai, J. Phys. Condens. Matter 4, 6039 (1992).

[7] R. Evans, J. Phys. (Paris), (Colloq.) 41, C-8 (1980).

[8] S. Iaroli, F. Ercolessi, and E. Tosatti, Surf. Sci. 251, 951 (1991).

[9] Z.-h. Cai and S. A. Rice (unpublished).

[10] L. Bosio et al., J. Electrochem. Soc. 139, 2110 (1992).

[11] E. B. Flom et al., Science 260, 332 (1993).

[12] M. Hasegawa and T. Ichikawa, J. Phys. Condens. Matter 3, 2769 (1991).

[13] S. K. Lai, Proc. Natl. Sci. Counc., Repub. China, Part A: Phys. Sci. Eng. 15, 191 (1991).

[14] E. H. Kawamoto et al., Phys. Rev. B 47, 6847 (1993).

[15] O. M. Magnussen et al., Phys. Rev. Lett. 74, 4444 (1995).

[16] P. Ascarelli, Phys. Rev. 143, 36 (1966).

[17] A. Di Cicco and A. Filipponi, Europhys. Lett. 27, 407 (1994).

[18] X. S. Gong et al., Europhys. Lett. 21, 469 (1993).

[19] M. J. Regan et al. (to be published).

[20] P. S. Pershan and J. Als-Nielsen, Phys. Rev. Lett. 52, 759 (1984).

[21] A. Braslau et al., Phys. Rev. B 38, 2457 (1988).

[22] M. P. D'Evelyn and S. A. Rice, J. Chem. Phys. 78, 5081 (1983).

[23] D. Turnbull, in Physics of Non-Crystalline Solids, edited by J. A. Prins (North-Holland, Amsterdam, 1964), p. 41.

[24] F. Spaepen, in Solid State Physics, edited by H. Ehrenreich and D. Turnbull (Academic Press, Boston, 1994), Vol. 47, p. 1.

[25] E. B. Flom et al., J. Chem. Phys. 96, 4743 (1992).

[26] R. Trittibch, C. Grütter, and J.H. Bilgram, Phys. Rev. B 50, 2529 (1994). 